\title{
Emmanuelle Brault, Le tigre et le loup
}

\section{Simona Rossi}

\section{(2) OpenEdition}

\section{Journals}

\section{Edizione digitale}

URL: http://journals.openedition.org/studifrancesi/35366

DOI: 10.4000/studifrancesi.35366

ISSN: 2421-5856

\section{Editore}

Rosenberg \& Sellier

\section{Edizione cartacea}

Data di pubblicazione: 1 novembre 2005

Paginazione: 464

ISSN: 0039-2944

\section{Notizia bibliografica digitale}

Simona Rossi, «Emmanuelle Brault, Le tigre et le loup», Studi Francesi [Online], 146 (XLIX | II) | 2005,

online dal 30 novembre 2015, consultato il 19 avril 2021. URL: http://journals.openedition.org/ studifrancesi/35366 ; DOI: https://doi.org/10.4000/studifrancesi.35366

\section{Questo documento è stato generato automaticamente il 19 avril 2021.}

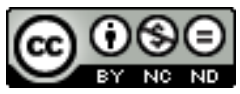

Studi Francesi è distribuita con Licenza Creative Commons Attribuzione - Non commerciale - Non opere derivate 4.0 Internazionale. 


\title{
Emmanuelle Brault, Le tigre et le loup
}

\author{
Simona Rossi
}

1 Emmanuelle Brault comincia la sua carriera di scrittrice con questo romanzo del tutto particolare, a metà tra la fantascienza e il racconto d'avventura, dove realtà e fantasia si mescolano in modo magistrale. La vicenda comincia nel quartiere cinese di una città somigliante a Montréal: qui una giovane di nome Michelle, ossessionata dalle tenebre malefiche che avvolgono il mondo $\mathrm{e}$ da un segreto che non verrà mai svelato, incontra Khaï, un orientale a capo di un gruppo di fanatici che sposano il supremo obiettivo di riportare la pace nel loro paese d'origine, devastato dalla guerra. Tra Khaï e la giovane si stabilisce da subito una particolare sintonia e quando lui si rende conto che sono in grado di leggere l'uno nei pensieri dell'altra, le chiede di unirsi al suo gruppo. In realtà Khaï ha riconosciuto in lei l'incarnazione del Lupo, colui che unendosi alla Tigre rivelerà l'Imponderabile e comincerà una nuova stirpe, portando pace e prosperità. Gli avvenimenti successivi all'ingresso di Michelle nel gruppo, così, sono relativi all'iniziazione di quest'ultima, che attraverso la lettura di scritture magiche e prove di coraggio, deve imparare a controllare e gestire i suoi poteri paranormali. È proprio Michelle a raccontare in prima persona ciò che le accade, anche se talvolta la narrazione della donna s'interrompe bruscamente per dare la parola a Khaï, producendo un forte effetto di rottura. L'intero romanzo è costruito sul frammento, del resto, sull'imprecisione e sul capovolgimento continuo, dunque il lettore deve essere sempre pronto a volgere lo sguardo altrove, basti pensare che ad un certo punto la vicenda si sposta fisicamente dalla città che somiglia a Montréal ad un paese che somiglia alla Cambogia. Tale principio di «verosimiglianza», tra l'altro, per cui ciò che appare finto potrebbe essere vero e ciò che invece sembra vero potrebbe non esserlo, contribuisce ad alimentare l'alone di mistero ed esoterismo che serpeggia nell'intero testo, lasciando il lettore completamente disorientato, privo dei suoi abituali punti di riferimento e per di più alle prese con una schiera di simboli tutti da interpretare.

2 Fatto di realtà parallele, rivelazioni oscure e personaggi che appaiono e scompaiono all'improvviso, senza che si riesca in qualche modo a dar loro un'identità precisa, Le tigre et le loup non è certo un'oasi sicura, quindi, bensì una sorta di mondo sospeso nel vuoto, dove per capire, o anche solo per tentare di capire, non è sufficiente leggere, bisogna aprire la mente all'Inconnu ed essere disposti ad accettarlo. A ben rifletterci, 
infatti, tutta la vicenda ruota attorno ad un incontro che deve avvenire tra due entità molto diverse tra loro: il Lupo, che compare a Montréal e potrebbe incarnare dunque il simbolo della cultura occidentale, e la Tigre, cambogiana, simbolo forse della cultura orientale. Naturalmente l'autrice non impone nessuna chiave di lettura, ma in compenso ci regala un sorprendente happy end: Michelle, presa coscienza del suo ruolo di restauratrice della Terre Vénérée, incontra la Tigre e genera con lei un bambino, segno evidente della pace ritrovata e della nuova fecondità della terra. Opera a dir poco labirintica, ricca di risvolti interessanti tutti da scoprire, Le tigre et le loup rappresenta senza dubbio una sfida stimolante per il lettore. 